MODELING, IDENTIFICATION AND CONTROL, 1990, VOL. 11, NO. 3, 141-154

doi:10.4173/mic.1990.3.2

\title{
Mechanical networks model for the truss system in structural engineering
}

\begin{abstract}
KESHENG WANG and ØYVIND BJØRKE
Keywords: Graph theory, system theory, networks, design theory, truss system, structure analysis.

This paper demonstrates how to use a mechanical networks model to analyse a three-dimensional truss system in structural engineering. A three-dimensional numerical example with a complete program written in the APL programming language is presented. The principles and concepts of this method are easily understood, and the formulation and computation of the problem can be carried out systematically and consistently. The main purpose is to develop a uniform, systematic theory and method which can be employed in the design process to analyse and synthesise a great number of engineering or non-engineering systems, such as mechanical, electrical, economic systems. The mechanical networks model presented in this paper has proved to be ideal for this type of analysis.
\end{abstract}

\section{Introduction}

In recent years, there has been a rapid growth in interest in the basic theoretical and methodological issues in the design process, which consists of two phases: analysis and synthesis of systems. An important phase in the design process is the synthesis of a system from elements in order to obtain a given performance. It is well known that numerous solutions to any specific problem are possible: that is, it is possible to create several alternative designs, each involving different systems of elements, to satisfy the design criteria. The final design is chosen among all possible designs by analysis, through a process of elimination. System synthesis, on the other hand, is largely an art, that obtains considerable assistance from the analysis of systems already designed. The most important matter is to develop a uniform, systematic analysis theory and method which can be employed in the design process to treat a great number of engineering or non-engineering systems, such as mechanical, electrical and economic systems. The mechanical networks model presented in this paper has proved to be this kind of ideal analysis method.

The development of the mechanical networks model has paved the way for the analysis of three-dimensional truss systems based on the directed linear graph theory. We have described the principles and concepts of the mechanical networks model theoretically in the literature (Wang and Bjørke (1989 b)). We can now demonstrate how the theory can be applied to solve a practical engineering problem. Truss systems, one of the basic problems in structural engineering, are taken as an example to show the validity of the mechanical network model. We have analysed the two-dimensional truss systems previously and details can be reviewed in these references (Bjørke and Wang

Received August 1, 1990.

Division of Production Engineering, Norwegian Institute of Technology, N-7034 Trondheim, Norway. 
(1990), Bjørke (1988), Wang and Bjørke (1990)). Here we go further and analyse threedimensional truss systems and show how to separate the formulation of the topological properties from the geometrical properties of the truss system, so as to facilitate their final combination. A set of functions has been developed for the automatic computer program. In order to simplify the problem, the paper is restricted to the static and linear analysis of trussed structures, although the theory discussed have a considerably wider scope of application, including static and dynamic, linear and nonlinear analysis.

\section{Representation of a truss system}

Figure 1 shows a schematic diagram of a simple three-dimensional truss system which consists of four uniform members which are pinned together at node point 5 and to a rigid base at node points 1, 2, 3 and 4 . We assume that the stiffness of each member is equal to 1 and that a force $\mathrm{F}$ acts on node point 5 .

\section{Primitive systems}

According to the concept of mechanical networks model, a complete and whole system in a structure can always be separated into several disconnected elements which are independent in physical properties. These elements are called primitive systems. In other words, a primitive system is a physical element which has terminal pairs at which different measurements are made, that is, all measuring procedures (instruments) have, at least conceptually, two terminal points. A measurement therefore always has to be associated with a topological line having two boundary points coinciding with the points in which the instrument is connected.

We can use a directed line to model the members of the truss system as the primitive system shown in Fig. 2.

\subsection{Dual variables}

In a truss system, there are two different kinds of physical quantities to characterize the mechanical properties of the element: force, $F$, and corresponding displacement, $U$. In the mechanical network model we call the force an intervariable, $I$, and the displacement a transvariable, $E$.

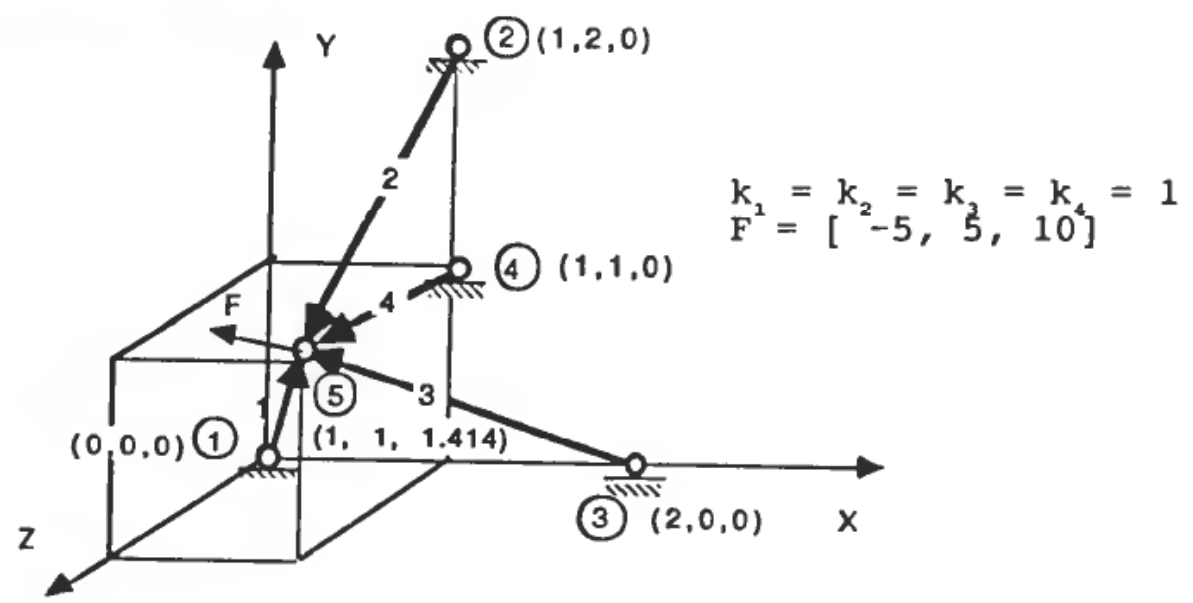

Figure 1. A three-dimensional truss system. 


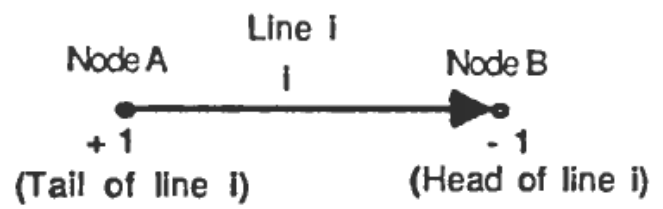

Figure 2. A graphical representation of the primitive system.
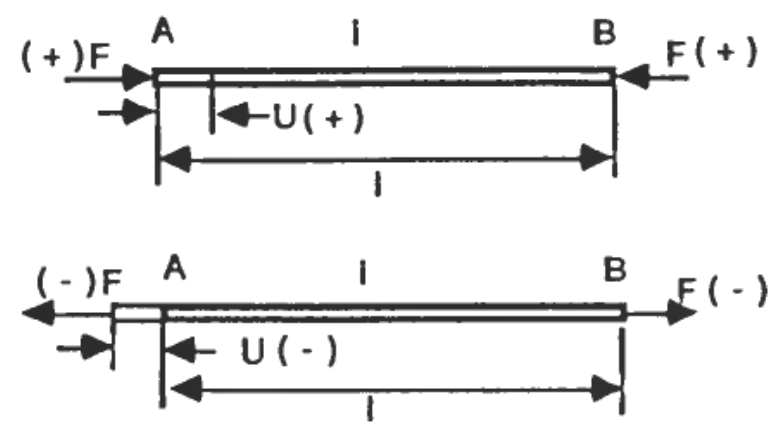

Figure 3. The sign definition of dual variables.

\subsection{Relationship between the dual variables}

Hoke's law can be used to relate the forces (transvariables) and displacements (intervariables) in a uniform truss member:

where

$$
F=(A E / l) U=k U
$$

$F$ force of the member

$U$ displacement of the member

$A$ cross-sectional area

$E$ elastic modulus

$l$ length of the element

$k$ stiffness of the element

Let us give the definition of the sign of a force (or the associated deformation) acting on a truss element as the following:

It is positive $(+)$, for compression (reduction).

It is negative (-), for tension (elongation).

In our mechanical networks model, we use the terminology intervariable, $i$, instead of forces, $F$, and transvariables, $e$, instead of displacements, $U$. For the primitive system, $i$, of a truss system, we can get

$$
i_{\mathrm{i}}=k_{\mathrm{i}} e_{\mathrm{i}}
$$

\subsection{Y-matrix}

Once the relationship of the element between the intervariable and transvariable has been determined, it is very easy to establish the whole relationship of the system 
between these dual variables which is called the Y-matrix. Assigning the given stiffness of each truss member, the Y-matrix is

$$
Y=\left[\begin{array}{llll}
k_{1} & & & \\
& k_{2} & & \\
& & k_{3} & \\
& & & k_{4}
\end{array}\right]=\left[\begin{array}{llll}
1 & & & \\
& 1 & & \\
& & 1 & \\
& & & 1
\end{array}\right]
$$

\section{Interconnected system}

The complete interconnected system is modeled by a directed graph which characterizes the topological properties of the system. The difference between electrical systems and the mechanical systems is that the variables in an electrical system are scalars, which are independent of the geometrical construction of the system, and the variables in a mechanical system are vectors, which are dependent on the geometrical construction.

That is the main reason why mechanical engineers never discovered the mechanical networks model in the mechanical engineering, but the electrical engineers have successfully developed the electrical networks theory in electrical engineering. Now it is time for mechanical engineering. Of course, it is a very difficult and laborious task. But if we can separate the formulation of topological properties from the geometrical properties of the system, then suddenly a mechanical system can be treated almost the same as an electrical system except that we need to superimpose the geometrical properties into the topological properties.

We are sure that the electrical networks can be considered a special case of the mechanical networks in which the geometrical transformation is set as zero. In this sense, the mechanical networks model is a more general analytic method than electrical networks. In this section we focus on the problem of how to separate the formulation of the topological properties from the geometrical properties of the truss system to determine the final combination.

\subsection{Topological properties}

\subsubsection{Behaviour graph}

By using of the graph theory, we can draw a behaviour graph to represent the interconnection of the element, which gives the topological properties of the truss system. The behaviour graph for the truss system is shown in Fig. 4.

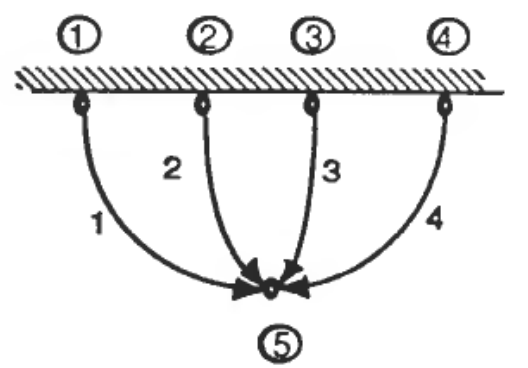

Figure 4. The behaviour graph of the truss system. 


\subsubsection{Topological matrix}

The behaviour graph enables us to directly obtain the corresponding topological matrices of the truss system. These are as follows:

$A U$-matrix

$$
\begin{gathered}
\text { lines } \\
A U=\frac{1}{3}\left[\begin{array}{rrrrr}
1 & 0 & 0 & 0 & -1 \\
0 & 1 & 0 & 0 & -1 \\
0 & 0 & 1 & 0 & -1 \\
0 & 0 & 0 & 1 & -1
\end{array}\right]
\end{gathered}
$$

$A$-matrix: (to remove the references: nodes 1, 2, 3 and 4)

$$
\begin{array}{ll}
\text { lines } & \multicolumn{2}{c}{\text { node }} \\
A= & \begin{array}{l}
1 \\
-1
\end{array}\left[\begin{array}{c}
-1 \\
-1 \\
-1 \\
-1
\end{array}\right]
\end{array}
$$

\subsection{Geometrical properties}

We note that in structural systems we often define the dual variables in two coordinate bases, one is the global coordinate system (node basis) and the other is the local coordinate system (line basis). These coordinate systems are shown in Fig. 5. In a truss system the node variables are measured in global coordinates and the element variables are measured in local coordinates.

\subsubsection{Transformation matrix}

Consider the typical three-dimensional truss member, $i$, which is shown in Fig. 5. The orientation of the member is defined by three angles $\alpha, \beta$ and $r$ with respect to the global coordinate axes. A transformation matrix, $T R$, will be used to represent the relationship between the global and local coordinate systems.
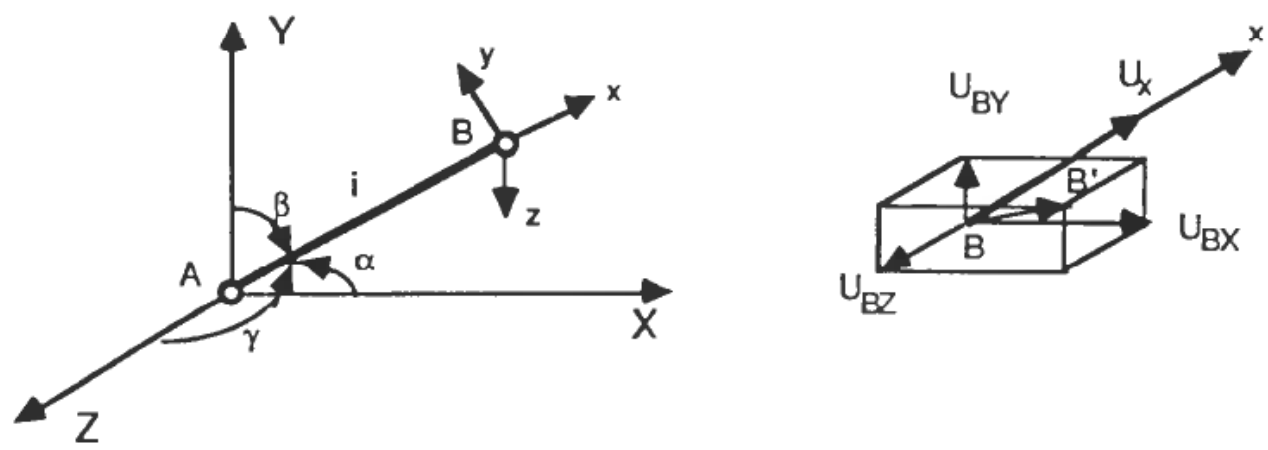

Figure 5. Global and local coordinates. 
In the local coordinates, the elementary displacement components $U_{y}$ and $U_{z}$ are of no interest for the truss element as they are perpendicular to element $i$ and do not cause a change in the length of the element. The elementary displacement component $U_{x}$ is in the axial direction of the element, and as it causes a change in the length of element $i$, it is of great interest in determining the force on the element. Therefore only the displacement $U_{x}$ has to be calculated into the transformation matrix $T R$ and it will make the form of the transformation matrix much simpler.

In order to unify the expressions in the mechanical networks model, we suggest that it is better to consider the two node points in element $i$ separately. The displacement of the node of the truss member in the global coordinate system $X-Y-Z$ can be transformed into the elongation of the truss element itself in local coordinate system $x-y-z$ by using of the transformation matrix $T R$.

Suppose the displacement vector of point $B$ (the head of arrow) is $U_{B}$ which is measured with respect to the global coordinates $X-Y-Z$, we can get the corresponding displacement vector of the element $i, U x$, which is measured with respect to the local coordinates $x-y-z$ as the following:

$$
\begin{aligned}
U_{x} & =\left(U_{B x} \cos \alpha+U_{B y} \cos \beta+U_{B z} \cos \gamma\right) \\
& =[-\cos \alpha-\cos \beta-\cos \gamma]\left[\begin{array}{l}
U_{B x} \\
U_{B y} \\
U_{B z}
\end{array}\right]
\end{aligned}
$$

The negative sign is obtained from the definition of the sign of the dual variables mentioned above, as we assume $U_{x}$ is the elongation of truss element in Fig. 5. The transformation matrix in the head point which is given -1 in the topological matrix is:

$$
T R_{i \text { (head) }}=\left[-\cos \alpha_{i}-\cos \beta_{i}-\cos \gamma_{i}\right]
$$

Using same principle and considering the definition of the sign, the transformation matrix for the tail point which is given +1 in the topological matrix is:

$$
T R_{i(\text { tail) }}=\left[\cos \alpha_{i} \cos \beta_{i} \cos \gamma_{i}\right]
$$

In this example, we can calculate the directional cosine for each element of the system from the configuration of the truss system shown in Fig. 1:

$$
\begin{array}{lll}
\cos \alpha_{1}=0.5, & \cos \beta_{1}=0.5, & \cos \gamma_{1}=0.707 \\
\cos \alpha_{2}=-0.5, & \cos \beta_{2}=0.5, & \cos \gamma_{2}=0.707 \\
\cos \alpha_{3}=0, & \cos \beta_{3}=-0.707, & \cos \gamma_{3}=0.707
\end{array}
$$

In the case of plane truss, $\gamma=0$ and only angles $\alpha$ and $\beta$ are considered. The transformation matrix is the same as the one given in (Bjørke and Wang (1990)), being as follows:

$$
T R_{i(\text { head) }}=\left[-\cos \alpha_{i}-\cos \beta_{i}\right]=\left[-\cos \alpha_{i}-\sin \alpha_{i}\right]
$$

and

$$
T R_{i(\text { tail) }}=\left[\begin{array}{lll}
\cos \alpha_{i} & \cos \beta_{i}
\end{array}\right]=\left[\begin{array}{lll}
\cos \alpha_{i} & \sin \alpha_{i}
\end{array}\right]
$$

where

$$
\alpha+\beta=90
$$




\subsubsection{Combined A-matrix}

The last step is to combine the topological and geometrical properties into a single combined $A$-matrix which is an incidence matrix for a mechanical network model.

$$
\begin{aligned}
A=T R * A & =\left[\begin{array}{ccc}
0.5 & 0.5 & 0.707 \\
-0.5 & 0.5 & 0.707 \\
0 & -0.707 & 0.707
\end{array}\right] *\left[\begin{array}{l}
-1 \\
-1 \\
-1
\end{array}\right] \\
& =\left[\begin{array}{ccc}
-0.5 & -0.5 & -0.707 \\
0.5 & -0.5 & -0.707 \\
0 & 0.707 & -0.707
\end{array}\right]
\end{aligned}
$$

We introduce a special operational sign ' $*$ ' in order to carry out the transformation multiplication, which combines both the topological matrix and geometrical matrix into an $A$-matrix. Attention should be paid to ensure that the '*' is an operator for transformation multiplication, which is different from normal matrix multiplication.

\section{Sources}

The types of given sources in a truss system can be classified into four groups.

(1) Node intervariable sources $I_{N}$

The forces which are acting on the node points can be defined as the given node transvariables sources, $I_{N}$. These given intervariable sources are measured with respect to the global coordinates (reference base), see Fig. 6(a).

(2) Node transvariable sources $E_{N}$

The displacements by which nodes are forced to move from the original position to a new position can be defined as the given transvariables sources, $E_{N^{*}}$. The given transvariable sources are measured with respect to the global coordinates (reference base), see Fig. 6(b).

(3) Elementary intervariable sources $I$

The forces which are acting in the elements can be defined as the given intervariable sources, $I$. The given intervariable sources are measured with respect to the local coordinates (element base). A system of weights and frictionless pulleys in parallel with the element represents the given intervariable source which is shown in Fig. 7.

(4) Elementary transvariable sources $E$

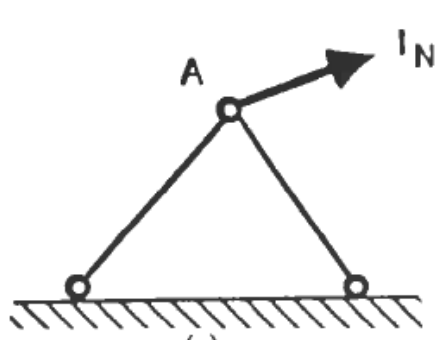

(a)

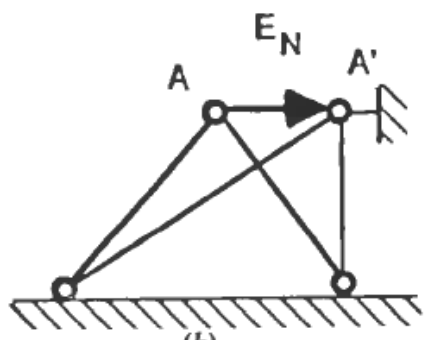

(b)

Figure 6. Node sources $I_{N}$ and $E_{N}$ in a truss system. 


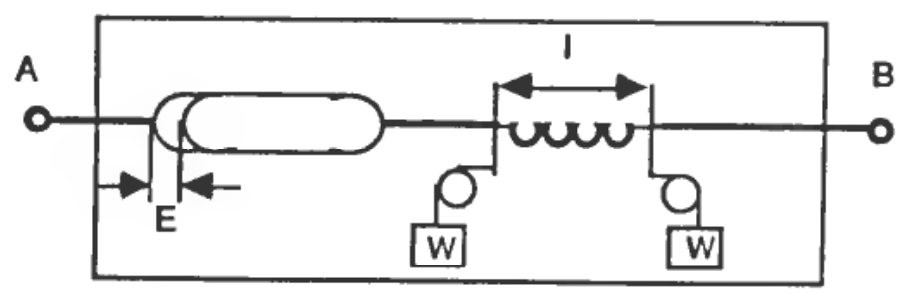

Figure 7. Element sources $I$ and $E$ in a truss element.

The displacements by which elements are forced to make elongation or reduction can be defined as elementary transvariable sources, $E$. A rigid turnbuckle in series with the element represents the given transvariable source which is shown in Fig. 7. In other cases, the displacement caused by the heating or cooling of the elements of a truss system can also be considered as the given transvariable sources.

In the mechanical networks model we can choose different methods to set up the formulation and obtain the solution of the problems. In general, we use the node method which corresponds to the stiffness method (displacement method) and the mesh method which corresponds to the flexibility method (force method) in structural problems. The four types of sources have to be transformed into one type of source during the formulation procedures. The details of this formulation procedure will be discussed in the next section.

\section{Formulation and solution}

Based on the Roth's diagram (Bjørke and Wang (1990)), we can easily get the formulation procedure for the truss system. First the node method is employed to solve the problem.

\subsection{The node method (stiffness method)}

(1) The given sources:

The force $F=\left[F_{x} F_{y} F_{z}\right]$ acting on node point 5 is the given course, which is defined as the node intervariable source $I_{N}$ of the system, see Fig. 1.

$$
I_{N}=\left[\begin{array}{lll}
-5 & 5 & 10
\end{array}\right]
$$

(2) $Y_{N}$-matrix

$$
Y_{N}=A^{t} Y A
$$

(3) The transvariable (displacement) in nodes

$$
e_{N}=Y_{N} I_{N}
$$

(4) The transvariable (displacement) in members

$$
e=A e_{N}
$$

(5) The intervariable (forces) in members

$$
i=Y e
$$




\subsection{The mesh method (flexibility method)}

If we employ the mesh method to solve the truss problem, the tree and cotree have to be defined. The number of the tree is exactly equal to the number of the staticdetermination, and the number of the cotree is the number of the redundant elements in the truss system.

(1) primitive system

$Z$ matrix:

$$
Z=(Y)^{-1}
$$

(2) Choose the trees and cotrees

$$
\begin{aligned}
& T(\text { tree })=123 \\
& L(\text { cotree })=4
\end{aligned}
$$

(3) Topological matrices

$C$-matrix:

$$
\begin{aligned}
C_{T} & =-\left(A_{T}^{t}\right)^{-1} A_{L}^{t} \\
C & =\left[\begin{array}{l}
C_{T} \\
C_{L}
\end{array}\right]
\end{aligned}
$$

$B$-matrix:

$$
\begin{aligned}
B_{T} & =\left(A_{T}^{t}\right)^{-1} \\
B & =\left[\begin{array}{c}
B_{T} \\
B_{L}
\end{array}\right]
\end{aligned}
$$

(4) The transformation of the given sources:

In the mesh method, the given sources have to be transformed into mesh sources in order to obtain the equivalent mesh sources, $E_{L}$. The given source in the system is the node intervariable source $I_{N}$ which is the force acting on node point 5 of the truss system.

$$
E_{L}=C^{\prime}\left(-Z\left(B I_{N}\right)\right)
$$

(5) The transvariable (displacement) in meshes

$$
\begin{gathered}
Z_{L}=C^{t} Z C \\
Y_{L}=\left(Y_{L}\right)^{-1} \\
i_{L}=Y_{L} E_{L}
\end{gathered}
$$

(6) The transvariable (displacement) in members

$$
i=C i_{L}
$$

(7) The intervariable (forces) in members

$$
i=Y e
$$

\section{Automatic computer programming}

A computer language system known as APL (A Programming Language) is introduced as a programming language for the mechanical network model. Though the 
Program list

The two programs which are called TRUSS $\triangle$ NODE and TRUSS $\triangle$ MESH can be executed on an IBM PC interactively. The terminal section is given below:

\section{(1) Node method}

\section{MAKE TRUSS NODE}

A COMPUTING OF A TRUSS SYSTEM

A NODE METHOD

A PRIMITIVE SYSTEM

A Y-MATRIX

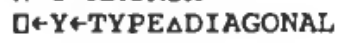

$\begin{array}{llll}1 & 1 & 1 & 1\end{array}$

$\begin{array}{llll}1 & 0 & 0 & 0\end{array}$

$\begin{array}{llll}0 & 1 & 0 & 0\end{array}$

$\begin{array}{llll}0 & 0 & 1 & 0\end{array}$

$0 \begin{array}{llll}0 & 0 & 0 & 1\end{array}$

A TOPOLOGICAL MATRICES

A A-MATRISE

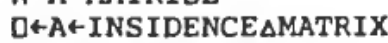

TYPE BRANIN TABLE

$123 \quad 34$

$\begin{array}{llll}5 & 5 & 5 & 5\end{array}$

$\begin{array}{lllll}1 & 0 & 0 & 0 & -1\end{array}$

$\begin{array}{lllll}1 & 1 & 0 & 0 & -1\end{array}$

$\begin{array}{lllll}0 & 0 & 1 & 0 & -1\end{array}$

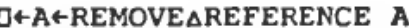

TYPE COLUMN-NUMBERS TO BE REMOVED

1234

$-1$

$-1$

$-1$

DETR\&TYPEAMATRIX

$0.5 \quad 0.50 .707$

$\begin{array}{llll}-0.5 & 0.5 & 0.707\end{array}$

$\begin{array}{llll}0 & -0.707 & 0.707\end{array}$

$\begin{array}{lll}0 & 0 & 1\end{array}$

$$
\begin{array}{ccc}
0.5 & 0.5 & 0.707 \\
-0.5 & 0.5 & 0.707 \\
0 & -0.707 & 0.707 \\
0 & 0 & 1
\end{array}
$$

TRNR 1111

$D \leftarrow A \leftarrow(A, T R N R)$ TRANS $\triangle M U L$ TR

$$
\begin{array}{ccc}
-0.5 & -0.5 & -0.707 \\
0.5 & -0.5 & -0.707 \\
0 & 0.707 & -0.707
\end{array}
$$

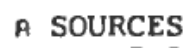

A SOURCES

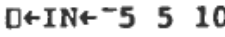

$-5510$

A FORMULATE AND SOLVE THE SYSTEM D-YN\&(TRA A) MUL Y MUL A

0.5000

$\begin{array}{lll}0 & 0.9998 & 0.2072\end{array}$

$\begin{array}{lll}0 & 0.20722 .5\end{array}$

ZN॰INV YN

D־eN־ZN MUL IN

$-104.2453 .649$

A RESULTS:

A DISPLACEMENTS IN ELEMENTS

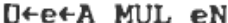

$\begin{array}{lllll}0.2978 & -9.702 & 0.4212 & -3.649\end{array}$

R FORCES ON ELEMENTS

Dњ1+Y MUL $\mathrm{C}$

$\begin{array}{lllll}0.2978 & -9.702 & 0.4212 & -3.649\end{array}$ 
(2) Mesh method $D \leftarrow Z \leftarrow I N V Y$
1000
$\begin{array}{lllll}0 & 1 & 0 & 0\end{array}$
$0 \begin{array}{llll}0 & 0 & 1 & 0\end{array}$
$\begin{array}{llll}0 & 0 & 0 & 1\end{array}$

A MESH METHOD

MAKE TRUSS $\triangle M E S H$

A PRIMITIVE SYSTEM

A Y-MATRIX IS SAME AS THE NODE METHOD

A TOPOLOGICAL MATRICES

A A-MATRIX IS SAME AS THE NODE METHOD

A CHOSE TRES

$D+T+123$

123

A C-MATRIX

D+C T MAKE $\triangle$ CAFROMAA A

$-0.4143$

$-0.4143$

$-0.5859$

1

D־B-T MAKE $\triangle B \triangle F R O M A A$

$\begin{array}{ccc}-1 & -0.4143 & -0.4143 \\ 1 & -0.4143 & -0.4143 \\ 0 & 0.8285 & -0.5859 \\ 0 & 0 & 0\end{array}$

A SOURCE

D+EL (TRA C) MUL $-Z$ MUL B MUL IN

$-6.154$

A FORMULATE AND SOLVE THE SYSTEM

$D+Z L+(T R A C)$ MUL $Z$ MUL $C$

1.687

D\&YL4INV ZL

0.5929

D 1 - + YL MUL EL

$-3.649$

A RESULTS:

A FORCES ON ELEMENTS

D+1 $+C$ MUL $1 L$

$\begin{array}{llll}1.512 & 1.512 & 2.138 & -3.649\end{array}$

D+1 tot $+1+$ B MUL IN

$\begin{array}{lllll}0.2978 & -9.702 & 0.4212 & -3.649\end{array}$

A DISPLACEMENT IN ELEMENTS

Detotez MUL itot

$\begin{array}{llll}0.2978 & -9.702 & 0.4212 & -3.649\end{array}$ 
language was not developed specifically for networks computation, it is in fact very suitable for the purpose. This is because:

(1) APL was designed at the outset to handle scalars, vectors, matrices, and rectangular arrays in any number of dimensions, either numerical arrays or characters. Many basic operations can be specified for arrays just as well as for scalars, without any loop written in the program. Programs in APL therefore tend to contain few loops. The user is encouraged to think of array operations without a logically irrelevant internal sequence; this is aesthetically pleasing and often illuminating.

(2) There is a high degree of consistency in APL, resulting from a high degree of generality in the definitions. Syntax is governed ruthlessly by a few simple rules. Once the vocabulary is learned, the language is easy to remember. There is a remarkable absence of arbitrary features that require frequent reference to the manual. The language therefore has a peculiar dignity and reasonableness.

(3) Because character arrays are first-class citizens, addressed as easily as a numerical array, scatterplots and other graphical displays can be programmed as readily as numerical calculations.

(4) The implementation of the language as a conversational computing system affords the flexibility that is badly needed but hard to find in special-purpose packages. It is easy for the user to change the data or compare the different results.

\subsection{Function list}

In order to develop a general software package, we have built up a series of functions which can be used to solve a great number of problems in different fields. These functions are written in APL and run on an IBM PC. With the help of the functions, it is very convenient and fast to write the program even if one does not know very much about the details of APL. The functions mainly consist of three groups: reading of data, topological matrices and computing operations. Some of the functions which are used for the truss system in structural engineering are listed as follows:

(1) Reading of data:

TYPE $\triangle$ MATRIX

TYPE $\triangle$ DIAGONAL

(2) Topological matrices:

\section{INCIDENCE $\triangle$ MATRIX \\ REMOVE $\triangle$ REFERENCE \\ MAKE $\triangle$ FROM $\triangle A$}

(3) Computing operations

TRA

INV

MUL

TRANS $\triangle$ MUL

COS

SIN

The details about the functions can be found in our exercise guidance (Wang and Bjørke (1990)). 


\section{Conclusions}

A mechanical network model has been successfully used to analyse a simple threedimensional truss system. The most important contribution here is to reveal the difference between electrical networks and mechanical networks. We have used a truss system to describe how first to separate the topological from the geometrical properties of the three-dimensional truss system, and then to combine them into a single $A$-matrix which leads to uniform, systematic formulation and computation of the given problem. We can say that electrical networks which only contain the topological properties are a special case of mechanical networks which contain both topological and geometrical properties.

The general procedure by which the mechanical networks model can be used to analyse the truss system in structure engineering is: (1) to establish the primitive system; (2) to determine the topological matrices; (3) to find the geometrical transformation matrix; (4) to obtain the combined matrices; (5) to formulate and compute systems. It seems that this general procedure is suitable for numerous kinds of systems in different areas of engineering (Bjørke (1988), Wang and Bjørke (1989a)).

The method seems simpler than most of the well-established methods of analysing truss systems (Beaufait, Rowan, Hoadley and Hackett (1970), Fleming (1989)). It is a great advantage that there is no increased difficulty in applying the procedure to more complex systems. Changes in loads, dimensions, and so forth, are most easily accommodated, because they only affect the input data and the fundamental procedure of formulation and solution is exactly the same. The method is also very user-friendly and convenient as the user executes the interactive computer program by a series of functions. The application of a mechanical networks model to three-dimensional frame systems and non-linear structural systems will be published later.

There are four kinds of methods in the mechanical network model: Node method, mesh method, region method and cut-set method. In this paper, we have employed two methods (node method and mesh method) to demonstrate the analytic procedure. Which method is best for analysing a given system depends on many factors. One of these is that we have to find the $Y_{N}$ or $Z_{L}$ is the simplest and it is possible to be inverted. We find that in the case of a truss system $Z_{L}$ is always the simplest. Another factor is that we have to see the kinds of sources that are given. We can choose the method which needs less transformation of sources. For example, in cases where $I_{N}$ is given, we prefer to use the node method rather than the mesh method.

The author's experience with the mechanical networks model has indicated that it is a uniform and systematic analytic method for the design process.

\section{REFERENCES}

Beaufait, F. W., Rowan, W. H., Hoadley, P. G. and Hackett, R. M. (1970). Computer Methods of Structural Analysis (Prentice-Hall, Inc.).

BJøRKE, Ø. and WANG, K. (1990). Maskinteknisk Systemteori, Lecture notes, Norwegian Institute of Technology, Trondheim, Norway.

BJØRKE, Ø. (1988). Toward a manufacturing systems theory - applications so far, Proceedings of the conference manufacturing international ' 88 ', Atlanta, Georgia. April, 1988.

Fleming, J. F. (1989). Computer Analysis of Structural Systems (McGraw-Hill Book Company).

WANG, K. and BJGRKE, Ø. (1989). The application of manufacturing system theory (MST) to dynamics of a rigid-body system, Proceedings of the international conference on modeling and simulation for optimization of manufacturing systems design and application, Arizona, USA. pp. 101-113, 8-10 November, 1989. 
WANG, K. and BJøRKE, Ø. (1989). The validity of mechanical network, International conference on systems science, Poland. September 1989.

WANG, K. and BJøRKE, Ø. (1990). Øvingene av maskinteknisk systemteori, Exercise guidance, Norwegian Institute of Technology, Trondheim, 1990. 